\begin{tabular}{|c|l|}
\hline Title & Kinetic boundary conditions for vapor-gas binary mixture \\
\hline Author(s) & Kobay ashi, Kazumichi; Sasaki, Kiyofumi; Kon, Misaki; Fujii, Hiroyuki; W atanabe, Masao \\
\hline Citation & $\begin{array}{l}\text { Microfluidics and Nanofluidics, 21/3), 53 } \\
\text { https:/doi.org/10.1007/s10404_017-1887-6 }\end{array}$ \\
\hline Issue Date & 2017-03-07 \\
\hline Doc URL & http://hdl.handle.net/2115/68447 \\
\hline Rights & The final publication is available at Springer via http://dx.doi.org/10.1007/s10404-017-1887-6 \\
\hline Type & article (author version) \\
\hline File Information & kobayashi_rev.pdf \\
\hline
\end{tabular}

Instructions for use 


\title{
Kinetic boundary conditions for vapor-gas binary mixture
}

\author{
Kazumichi Kobayashi, Kiyofumi Sasaki, \\ Misaki Kon, Hiroyuki Fujii, Masao \\ Watanabe
}

Received: date / Accepted: date

\begin{abstract}
Using molecular dynamics simulations, the present study investigated the precise characteristics of the binary mixture of condensable gas (vapor) and non-condensable gas (NC gas) molecules creating kinetic boundary conditions (KBCs) at a gas-liquid interface in equilibrium. We counted the molecules utilizing the improved two-boundary method proposed in previous studies by Kobayashi et al. (Heat Mass Trans 52: 1851-1859. doi:10.1007/s00231015-1700-6, 2016). In this study, we employed Ar for the vapor molecules, and Ne for the NC gas molecules. The present method allowed us to count easily the evaporating, condensing, degassing, dissolving, and reflecting molecules in order to investigate the detailed motion of the molecules, and also to evaluate the velocity distribution function of the KBCs at the interface. Our results showed that the evaporation and condensation coefficients for vapor and $\mathrm{NC}$ gas molecules decrease with the increase of the molar fraction of the NC gas molecules in the liquid. We also found that the KBCs can be specified as a function of the molar fraction and liquid temperature. Furthermore, we discussed the method to construct the KBCs of vapor and NC gas molecules.
\end{abstract}

Keywords Kinetic boundary conditions - Evaporation and condensation . Binary mixture

\section{Introduction}

In recent years, with the progress of advanced technologies using net evaporation/condensation, heat and mass transfer between liquid and gas phases

K. Kobayashi

Division of Mechanical and Space Engineering, Hokkaido University, Kita 13 Nishi 8, Kitaku, Sapporo, Hokkaido, 060-8628 Japan

Tel.: +81-11-7066429

Fax: +81-11-7066429

E-mail: kobakazu@eng.hokudai.ac.jp 
has become very important from the viewpoints of molecular and micro-scale mass transfer [Wörner (2012); Lee et al. (2014); Lu et al. (2015)]. Generally, the gas phase adjacent to the liquid phase is composed of a multicomponent mixture of molecules, e.g., condensable gas molecules and non-condensable gas molecules, hereafter referred to as vapor and $N C$ gas molecules, respectively. The influence of $\mathrm{NC}$ gas molecules on the evaporation and condensation of vapor molecules or the dissolution of NC gas in the liquid phase (Henry's law) has received much attention in the physical processes of heat and mass transfer at a gas-liquid interface, for example, in cavitation bubble collapse and molecular distillation with evaporation and condensation [Matsumoto and Takemura (1994); Kreider et al. (2011); Lee et al. (2014)].

In general, a gas phase in the vicinity of a gas-liquid interface during net evaporation/condensation is a non-equilibrium region. Molecular gas dynamics (MGD) based on the molecular velocity distribution function, which is governed by the Boltzmann equation, is capable of describing gas flows in the vicinity of the interface. Thus, gas flows composed of a vapor and NC gas mixture have been studied and useful results have been obtained by solving the Boltzmann equation [Aoki et al. (1998); Takata et al. (2003); Taguchi et al. (2003)]. The vapor and NC gas flow is described using two velocity distribution functions of molecules in the analysis of MGD for a binary mixture : $f^{V}(\boldsymbol{x}, \boldsymbol{\xi}, t)$ is the velocity distribution function for vapor molecules, and $f^{G}(\boldsymbol{x}, \boldsymbol{\xi}, t)$ is the velocity distribution function for NC gas molecules, where $\boldsymbol{x}(=x, y, z)$ is the physical coordinate, and $\boldsymbol{\xi}\left(=\xi_{x}, \xi_{y}, \xi_{z}\right)$ gives the molecular velocities along the $x, y$, and $z$ physical axes, and $t$ is time.

The kinetic boundary condition (KBC) at the gas-liquid interface for the Boltzmann equation plays an essential role in the region near the interface. The $\mathrm{KBC}$ for vapor molecules in weak non-equilibrium has been given as follows [Kon et al. (2014)]:

$$
f_{\text {out }}^{V}=\frac{\alpha_{\mathrm{e}} \rho^{*}+\left(1-\alpha_{\mathrm{c}}\right) \sigma^{V}}{\left(\sqrt{2 \pi R^{V} T_{L}}\right)^{3}} \exp \left(-\frac{\xi_{i}^{2}}{2 R^{V} T_{L}}\right), \text { for } \xi_{z}>0,
$$

where $f_{\text {out }}^{V}$ is the velocity distribution function of outgoing vapor molecules from the interface to the vapor phase. $\rho^{*}\left(T_{L}\right)$ is the saturated vapor density at the liquid temperature $T_{L} . R^{V}$ is the gas constant of vapor. In this paper, a positive $\xi_{z}$ value refers to molecular velocity from the interface to the vapor phase, where $z$ is the direction normal to the interface and $x$ and $y$ are tangential to the interface. Additionally, $\xi_{i}^{2}$ is given by $\xi_{i}^{2}=\xi_{x}^{2}+\xi_{y}^{2}+\xi_{z}^{2}$. The definition of $\sigma^{V}$ is written as

$$
\sigma^{V}=-\sqrt{\frac{2 \pi}{R^{V} T_{L}}} \int_{-\infty}^{0} \int_{-\infty}^{\infty} \int_{-\infty}^{\infty} \xi_{z} f_{\text {coll }}^{V} \mathrm{~d} \xi_{x} \mathrm{~d} \xi_{y} \mathrm{~d} \xi_{z},
$$

where $f_{\text {coll }}^{V}$ is the velocity distribution function of colliding vapor molecules from the vapor phase to the interface. The velocity distribution function of the entire molecular velocity space of the vapor at the interface, $f^{V}$, is $f^{V}=$ $f_{\text {coll }}^{V}+f_{\text {out }}^{V} \cdot \alpha_{\mathrm{e}}$ and $\alpha_{\mathrm{c}}$ in Eq.(1) are the so-called evaporation and condensation 
coefficients, respectively. From our previous study [Kon et al. (2014, 2016a)], Eq. (1) is defined only by the liquid temperature.

The KBC for the NC gas molecules has been used to define the diffuse reflection of molecules, which is given as

$$
f_{\text {out }}^{G}=\frac{\sigma^{G}}{\left(\sqrt{2 \pi R^{G} T_{L}}\right)^{3}} \exp \left(-\frac{\xi_{i}^{2}}{2 R^{G} T_{L}}\right), \text { for } \xi_{z}>0
$$

where $R^{G}$ is the gas constant of NC gas, and

$$
\sigma^{G}=-\sqrt{\frac{2 \pi}{R^{G} T_{L}}} \int_{-\infty}^{0} \int_{-\infty}^{\infty} \int_{-\infty}^{\infty} \xi_{z} f_{\text {coll }}^{G} \mathrm{~d} \xi_{z} \mathrm{~d} \xi_{y} \mathrm{~d} \xi_{z}
$$

In this equation, all of the $\mathrm{NC}$ gas molecules coming into the liquid from the gas phase are reflected at the interface.

For the KBC of vapor (Eq. (1)), several studies have been carried out to determine the rate of evaporation and reflection of molecules based on molecular simulations in the case of single-component system (for example, see the references by Frezzotti (2011a), Kryukov and Levashov (2015), Xie et al. (2012), and our studies in Kobayashi et al. (2016b) and Kon et al. (2014, 2016a)). Furthermore, Frezzotti (2011b) performed molecular dynamics (MD) simulations of binary mixtures for Ar-Kr vapors using the concept of spontaneous evaporation [Ishiyama et al. (2004)], and obtained the values of evaporation coefficients for $\mathrm{Ar}$ and $\mathrm{Kr}$ molecules. However, there are no studies that consider the influence of $\mathrm{NC}$ gas molecules on the evaporation and condensation of vapor molecules with experimental and theoretical methods. Therefore, the KBCs in the case of the binary-mixture problem composed of $\mathrm{NC}$ gas and vapor molecules have not been constructed. Additionally, for the KBC of NC gas (Eq. (3)), the dissolution of NC gas molecules is not considered; Eq. (3) cannot satisfy the Henry's law.

Recently, the authors proposed a method to construct the KBC by counting the evaporating, reflecting, and condensing vapor molecules for a singlecomponent system [Kobayashi et al. (2016b)]. This method uses the vapor and liquid boundaries. The concept of using two boundaries was proposed by Meland et al. (2004) and Gu et al. (2010a); we improved the method incorporating the concept of spontaneous evaporation [Ishiyama et al. (2004)]. Furthermore, the authors carried out an equilibrium MD simulation using the improved method, and showed that this method is capable of constructing the KBC for the nonequilibrium single-component and equilibrium binary mixture problems [Kon et al. (2016b); Kobayashi et al. (2016a)]. Thus, the aim of this study is to develop KBCs for the vapor-NC gas binary mixture in equilibrium by changing both the molar fraction of gas in the liquid and the temperature. In this paper, we utilize $\mathrm{Ar}$ as vapor molecules and $\mathrm{Ne}$ as gas molecules. 


\section{New KBCs for vapor and NC gas molecules}

\subsection{Definition of KBCs for binary mixture}

To define the KBCs for a binary mixture, we consider the ten mass fluxes at the interface composed of vapor and $\mathrm{NC}$ gas molecules as shown in Fig. 1. For vapor molecules, evaporating molecules and condensing molecules are considered. Also, for NC gas molecules, degassing molecules and dissolving molecules are considered. For these molecules, we propose new KBCs for binary mixture as alternatives to Eqs. (1) and (3), using not only the liquid temperature but also the molar fraction of $\mathrm{NC}$ gas molecules in liquid to consider the influence the NC gas molecules on phase change of vapor molecules and to satisfy the Henry's law as follows:

$$
\begin{aligned}
f_{\text {out }}^{V} & =\frac{\alpha_{\mathrm{e}}^{V}\left(\phi, T_{L}\right) \rho^{V *}\left(\phi, T_{L}\right)+\left[1-\alpha_{\mathrm{c}}^{V}\left(\phi, T_{L}\right)\right] \sigma^{V}}{\left(\sqrt{2 \pi R^{V} T_{L}}\right)^{3}} \\
& \times \exp \left(-\frac{\xi_{i}^{2}}{2 R^{V} T_{L}}\right), \\
f_{\text {out }}^{G} & =\frac{\alpha_{\mathrm{e}}^{G}\left(\phi, T_{L}\right) \rho^{G *}\left(\phi, T_{L}\right)+\left[1-\alpha_{\mathrm{c}}^{G}\left(\phi, T_{L}\right)\right] \sigma^{G}}{\left(\sqrt{2 \pi R^{G} T_{L}}\right)^{3}} \\
& \times \exp \left(-\frac{\xi_{i}^{2}}{2 R^{G} T_{L}}\right),
\end{aligned}
$$

where $\phi$ is the molar fraction in the liquid, which is defined as:

$$
\phi=\frac{N^{G}}{N^{V}+N^{G}},
$$

where $N^{G}$ is the number of $\mathrm{NC}$ gas molecules dissolving in liquid and $N^{V}$ is the number of vapor molecules in bulk liquid phase, respectively. $\rho^{V *}$ is the saturated vapor density, and $\rho^{G *}$ is the $\mathrm{NC}$ gas density at the equilibrium state. $\alpha_{\mathrm{e}}^{V}$ and $\alpha_{\mathrm{c}}^{V}$ are the evaporation and condensation coefficients for vapor molecules, and $\alpha_{\mathrm{e}}^{G}$ and $\alpha_{\mathrm{c}}^{G}$ are the degassing and dissolution coefficients for $\mathrm{NC}$ gas molecules, respectively. Hereafter, we call the degassing coefficient the "evaporation coefficient" and the dissolution coefficient the "condensation coefficient" for gas molecules, the same as for the vapor molecules. Also, $f_{\text {out }}^{V}$ and $f_{\text {out }}^{G}$ are rewritten as

$$
f_{\text {out }}^{V}=f_{\mathrm{e}}^{V}+f_{\mathrm{r}}^{V},
$$

and

$$
f_{\text {out }}^{G}=f_{\mathrm{e}}^{G}+f_{\mathrm{r}}^{G},
$$

where $f_{\mathrm{e}}^{V}$ is the velocity distribution function of evaporating vapor molecules, and $f_{\mathrm{r}}^{V}$ is that of reflecting vapor molecules. Additionally, $f_{\mathrm{e}}^{G}$ is the velocity distribution function of degassing $\mathrm{NC}$ gas molecules, and $f_{\mathrm{r}}^{V}$ is that of reflecting $\mathrm{NC}$ gas molecules. 
As shown in Eqs. (5) and (6), these variables are the function of not only the liquid temperature $T_{L}$ but also $\phi$. This concept was proposed in the previous study by Frezzotti (2011b). From the above definition, we can treat the degassing and dissolution of NC gas molecules in the bulk liquid. Similarly, it is well known that $\rho^{V *}$ and $\rho^{G *}$ are functions of $T_{L}$ and $\phi$ in the previous experiment by Streett $(1965,1967)$.

The definitions of $\alpha_{\mathrm{e}}^{V}, \alpha_{\mathrm{c}}^{V}, \alpha_{\mathrm{e}}^{G}$, and $\alpha_{\mathrm{c}}^{G}$ are as follows:

$$
\alpha_{\mathrm{e}}^{V}=\frac{J_{\mathrm{e}}^{V}}{J_{\text {out }}^{V *}}, \alpha_{\mathrm{c}}^{V}=\frac{J_{\mathrm{c}}^{V}}{J_{\text {coll }}^{V}}, \alpha_{\mathrm{e}}^{G}=\frac{J_{\mathrm{e}}^{G}}{J_{\text {out }}^{G *}}, \alpha_{\mathrm{c}}^{G}=\frac{J_{\mathrm{c}}^{G}}{J_{\text {coll }}^{G}},
$$

where, for vapor molecules, $J_{\mathrm{e}}^{V}$ is the evaporating molecular mass flux, $J_{\text {out }}^{V}$ is the outgoing molecular mass flux, $J_{\mathrm{c}}^{V}$ is the condensing molecular mass flux, and $J_{\text {coll }}^{V}$ is the colliding molecular mass flux. Additionally, for NC gas molecules, $J_{\mathrm{e}}^{G}$ is the degassing molecular mass flux, $J_{\text {out }}^{G}$ is the outgoing molecular mass flux, $J_{\mathrm{c}}^{G}$ is the dissolving molecular mass flux, and $J_{\text {coll }}^{G}$ is the colliding molecular mass flux, respectively. Superscript $*$ denotes the equilibrium state. The definitions $\alpha_{\mathrm{e}}^{V}$ and $\alpha_{\mathrm{c}}^{V}$ are the same as the single vapor component system. From the previous MD simulation of single component system [Meland et al. (2004)], the evaporation and condensation coefficients are not dependent on the molecular velocity tangential to the interface. Hence, the definition of condensation and evaporation coefficients is independent on the incident angle between the colliding molecular velocity and the interface. Using these definitions of the coefficients, we can investigate the influence of NC gas on the evaporation and condensation of vapor molecules, and consider the $\mathrm{NC}$ gas dissolution in the liquid.

In the vapor-liquid equilibrium state, the following relations are satisfied:

$$
\begin{aligned}
& \sigma^{V} \rightarrow \rho^{V *}, \sigma^{G} \rightarrow \rho^{G *}, J_{\mathrm{e}}^{V}=J_{\mathrm{c}}^{V}, J_{\mathrm{e}}^{G}=J_{\mathrm{c}}^{G}, \\
& J_{\text {out }}^{V}=J_{\text {coll }}^{V}, J_{\text {out }}^{G}=J_{\text {coll }}^{G}, \alpha_{\mathrm{e}}^{V}=\alpha_{\mathrm{c}}^{V}, \alpha_{\mathrm{e}}^{G}=\alpha_{\mathrm{c}}^{G} .
\end{aligned}
$$

In this study, we carry out an equilibrium MD simulation of $\mathrm{Ar}-\mathrm{Ne}$ molecules and determine whether $\rho^{V *}, \alpha_{\mathrm{e}}^{V}, \alpha_{\mathrm{c}}^{V}, \rho^{G *}, \alpha_{\mathrm{e}}^{G}$, and $\alpha_{\mathrm{c}}^{G}$ are functions of $T_{L}$ and $\phi$ in the vapor-liquid equilibrium state.

\subsection{Equilibrium simulation}

In this section, we describe a method for gas-liquid equilibrium MD simulations for Ar-Ne molecules. A system composed of 6000 Ar molecules was considered in a simulation box with dimensions $L_{x} \times L_{y} \times L_{z}$ where $L_{x}=7$ $\mathrm{nm}, L_{y}=7 \mathrm{~nm}$, and $L_{z}=35 \mathrm{~nm}$ (see Fig. 2). At the center of the $z$ axis $\left(L_{z}=17.5 \mathrm{~nm}\right)$, a plane liquid film is formed. Periodic boundary conditions were imposed on the simulation system edges in all three dimensions. The system is symmetric from the center of the system. Therefore, we analyzed the molecular behavior by replicating the simulation box. By changing the number of Ne molecules, we adjusted the molar concentration in the liquid. 
The numbers of Ne molecules were 0,600, 1200, 1800, 2400, and 3000. As the number of $\mathrm{Ne}$ molecules increases, the value of $\phi$ increases.

For the intermolecular potential of Ar-Ar, Ar-Ne, and Ne-Ne molecules, a 12-6 type Lennard--Jones potential was used:

$$
\varphi(r)=4 \epsilon\left[\left(\frac{a}{r}\right)^{12}-\left(\frac{a}{r}\right)^{6}\right],
$$

where the lengths of Ar-Ar and Ne-Ne, $a_{A r-A r}$ and $a_{N e-N e}$, are $3.405 \AA$ and $2.750 \AA$, respectively, and the potential depths of Ar-Ar and Ne-Ne, $\epsilon_{A r-A r} / k$, and $\epsilon_{N e-N e} / k$, are $119.8 \mathrm{~K}$ and $35.05 \mathrm{~K}$, respectively, where $k$ is the Boltzmann constant. For Ar-Ne molecules, $a$ and $\epsilon$ obey the following Lorentz-Berthelot law :

$$
\begin{aligned}
& a_{A r-N e}=A \frac{a_{A r-A r}+a_{N e-N e}}{2}, \\
& \epsilon_{A r-N e}=B \sqrt{\epsilon_{A r-A r} \cdot \epsilon_{N e-N e}},
\end{aligned}
$$

where $A=1.01903$ and $B=0.89554$ are the constants used to satisfy the Henry's law (dissolution of Ne molecules in Ar liquid) [Baidakov and Protsenko (2008)]. Also, from the MD simulation by Baidakov and Protsenko, they concluded that the above MD parameters can satisfy the phase diagrams of Ar-Ne equilibrium system obtained from the experiment. The Newton's equations of motion for the Ar and Ne molecules in the system were solved using the leap-frog method. The cutoff radius was set to $15 \AA$. When the steady state was attained, a liquid phase was formed at the center of the system.

The initial condition was obtained from the temperature control simulation [Hansen and McDonald (1990)] for the system. After the system reached the vapor-liquid equilibrium state, we performed an equilibrium simulation without temperature control. The time increment was $5 \mathrm{fs}$; the sampling period was 1 ps (i.e., 200 time steps). To obtain the precise values of the macroscopic variables and the velocity distribution function, the simulation continued for $200 \mathrm{~ns}$. In this study, we performed the equilibrium simulations at $85 \mathrm{~K}$ and $95 \mathrm{~K} .85 \mathrm{~K}$ is the low temperature case to satisfy the ideal gas law in gas phase [Ishiyama et al. (2004)], and $95 \mathrm{~K}$ is the slightly high temperature case.

\subsection{Method for counting molecular mass fluxes}

In this simulation, we used the two-boundary method proposed by Kobayashi et al. (2016b). The concept of using two boundaries was discussed by Meland et al. (2004) and Gu et al. (2010a). As shown in Fig. 3, the NC gas and liquid phases contain the gas and liquid boundaries, respectively. The region between the two boundaries is called the interphase. This method allows the counting of evaporating, condensing, reflecting, degassing, and dissolving molecules at the gas boundary. The definitions of both boundaries are important for counting molecular mass fluxes in MD simulations (Fig. 3). 
For vapor molecules, the evaporating molecular mass flux, $J_{\mathrm{e}}^{V}$, was computed using the number of molecules crossing from the liquid boundary to the gas boundary. Conversely, the condensing molecular mass flux, $J_{\mathrm{c}}^{V}$, was computed using the number of molecules crossing from the gas boundary to the liquid boundary. The reflecting molecular mass flux, $J_{\mathrm{r}}^{V}$, was computed from the number of molecules crossing the gas boundary twice, as shown in Fig. 3. These molecular mass fluxes were computed as follows

$$
J=\frac{1}{n_{\mathrm{s}}} \sum_{n_{\mathrm{s}}} \frac{m \Delta N}{S \Delta t}
$$

where $n_{\mathrm{s}}$ is the sampling number, $\Delta N$ is the number of molecules crossing the boundary during the time $\Delta t, m$ is the mass of a molecule, and $S$ is the cross-sectional area of the boundary. The outgoing molecular mass flux, $J_{\text {out }}^{V}$, and colliding molecular mass flux, $J_{\text {coll }}^{V}$, were computed using $J_{\text {out }}^{V}=J_{\mathrm{e}}^{V}+J_{\mathrm{r}}^{V}$ and $J_{\text {coll }}^{V}=J_{\mathrm{c}}^{V}+J_{\mathrm{r}}^{V}$, respectively. Using the same procedure, we can obtain the NC gas mass fluxes, $J_{\mathrm{e}}^{G}, J_{\mathrm{r}}^{G}$ and $J_{\mathrm{c}}^{G}$, respectively, as $J_{\text {out }}^{G}=J_{\mathrm{e}}^{G}+J_{\mathrm{r}}^{G}$ and $J_{\text {coll }}^{G}=J_{\mathrm{c}}^{G}+J_{\mathrm{r}}^{G}$. The temporal fluctuation for the evaporation molecular mass flux was discussed in the previous study [Kobayashi et al. (2016b)].

To utilize the above counting method, the positions of the gas and liquid boundaries are important. In the present study, we used the positions of gas and liquid boundaries determined from our previous study for single Ar components [Kobayashi et al. (2016b)]. In our simulations, the gas boundary was considered to be the position of the KBC for the Boltzmann equation. Here, we introduced the new coordinate:

$$
\bar{z}=\frac{z-Z_{\mathrm{m}}}{\delta}
$$

where $\delta\left(T_{L}, \phi\right)$ is the 10-90 thickness of the density transition layer. The thickness of $\delta$ was estimated using the following equation.

$$
\rho^{V}(z)=\frac{\rho_{\mathrm{B} g}^{V}+\rho_{\mathrm{B} l}^{V}}{2}+\frac{\rho_{\mathrm{B} g}^{V}-\rho_{\mathrm{B} l}^{V}}{2} \tanh \left(\frac{z-Z_{m}}{0.455 \delta}\right),
$$

where $\rho_{\mathrm{B} g}^{V}$ is the density of Ar molecules in the bulk gas phase, $\rho_{\mathrm{B} l}^{V}$ is that of Ar molecules in the bulk liquid phase, and $Z_{\mathrm{m}}$ is the center position of the density transition layers. In an equilibrium state, $\rho_{\mathrm{B} g}^{V}$ becomes the saturated vapor density, $\rho^{V *}$. This thickness is shown in Fig. 4 as a function of $T_{L}$ and $\phi$. These values were obtained from the equilibrium MD simulation. As can be seen from the figure, the thickness $\delta$ gradually increases with an increase of $\phi$. Additionally, as the temperature becomes larger, the value of $\delta$ increases. The temperature dependence of $\delta$ was reported from the previous MD simulation [Ishiyama et al. (2004)]. Using the new coordinate, the positions of the gas and liquid boundaries are determined to be $\bar{z}=3.0$ and $\bar{z}=-1.0$ from a previous study [Kobayashi et al. (2016b)]. In the previous study, we discussed about the influence of the position of liquid boundary for the evaporating molecular mass flux. 


\section{Results}

3.1 Macroscopic quantities and $\rho^{V *}$ and $\rho^{G *}$ in Eqs. (5) and (6)

First, we need to confirm that our MD simulation reaches the vapor-liquid equilibrium state. Figures 5(a) and (b) show the macroscopic quantities of $\mathrm{Ar}$ and Ne molecules in the vapor-liquid equilibrium state at $85 \mathrm{~K}$ and $95 \mathrm{~K}$, respectively. The abscissa is the normalized distance, $\bar{z}$. The ordinates show that $\rho^{V}$ and $\rho^{G}$ are the densities of $\mathrm{Ar}$ and $\mathrm{Ne}, T^{V}$ and $T^{G}$ are the temperatures of $\mathrm{Ar}$ and $\mathrm{Ne}$, and $v^{V}$ and $v^{G}$ are the velocities in z-direction of $\mathrm{Ar}$ and $\mathrm{Ne}$, respectively.

Around $\bar{z}=0$, there is a density transition layer of Ar. In the transition layer of Ar, the adsorption film of Ne molecules exists. This adsorption film was also observed in the previous MD simulations [Baidakov and Protsenko (2008)]. Furthermore, Ne molecules exist in the bulk Ar liquid, which leads to the dissolution of Ne molecules. The temperatures of $\mathrm{Ar}$ and Ne uniformly take the specified values in each simulation. Additionally, the gas velocities of $\mathrm{Ne}$ and $\mathrm{Ar}$ at $85 \mathrm{~K}$ and $95 \mathrm{~K}$ uniformly become zero. From the definitions of the mass fluxes relation at the gas-liquid interface [Kon et al. (2014)], the mass flux of vapor, $\rho^{V} v^{V}$, is defined as $\rho^{V} v^{V}=J_{\text {out }}^{V}-J_{\text {coll }}^{V}$. In the equilibrium state, $J_{\text {out }}^{V}$ is equal to $J_{\text {coll }}^{V}$ (see Tables 1-4). Hence, the value of $v^{V}$ becomes zero. This is important point for equilibrium. Same definition is applied to the NC gas molecules: from these results, we confirm that the system reaches the vapor-liquid equilibrium state.

Next, we investigate the vapor and NC gas densities, $\rho^{V *}$ and $\rho^{G *}$, as functions of the molar fraction $\phi$ (Fig. 6). $\rho^{V *}$ and $\rho^{G *}$ are obtained from the average values of $\rho^{V}$ and $\rho^{G}$ in the bulk gas phase, as shown in Fig. 5. These densities are included in KBCs (See Eqs. (5) and (6)). From Fig. 6(a), $\rho^{V *}$ at $95 \mathrm{~K}$ has a larger value than that at $85 \mathrm{~K}$. Additionally, we can see that the value of $\rho^{V *}$ increases with $\phi$. This increase with $\phi$ was observed in the experiments for the Ar-Ne mixture at the equilibrium state [Streett (1965, 1967)]. Furthermore, the values of $\rho^{G *}$ are approximately linear functions of $\phi$; the value of $\rho^{G *}$ becomes zero at $\phi=0$.

3.2 Molecular velocity distribution functions for vapor and $\mathrm{NC}$ gas molecules in Eqs. (8) and (9)

In this subsection, we show the velocity distribution functions of outgoing molecules related to the KBCs as shown in Eqs. (8) and (9). The normalized velocity distribution function of outgoing molecules in a vapor-liquid equilibrium state becomes the Maxwellian at the specified temperature. Using the present MD simulation, we confirm that the normalized velocity distribution functions, $\hat{f}_{\text {out }}^{V}$ and $\hat{f}_{\text {out }}^{G}$, are the Maxwellian at the liquid temperatures $(85 \mathrm{~K}$ and $95 \mathrm{~K}$ ) for any value of $\phi$. Hereafter, we investigate the velocity distribution functions, $f_{\mathrm{e}}^{V}, f_{\mathrm{r}}^{V}, f_{\mathrm{e}}^{G}$, and $f_{\mathrm{r}}^{G}$ in Eqs. (8) and (9). 
From the previous MD simulations in equilibrium, we confirmed that the velocity distribution function of evaporating and reflecting molecules in the tangential direction to the interface becomes the Maxwellian; on the other hand, that of the normal direction deviates from the Maxwellian [Kobayashi et al. (2016b)]. Therefore, we firstly show the velocity distribution function in the direction normal to the interface ( $z$ direction).

Figures 7(a) and (b) show the velocity distribution functions of evaporating and reflecting for Ar molecules (vapor) in the direction normal to the interface at $85 \mathrm{~K}$ as a function of $\phi$. In the present two-boundary method, the velocity distribution functions were measured using the plane perpendicular to the $z$ axis [Kobayashi et al. (2016b)]; evaporating and reflecting molecules only had positive velocities in the $z$ direction, and the results were presented as functions of $\zeta_{z} \hat{f}_{\mathrm{e}}^{V z}$ and $\zeta_{z} \hat{f}_{\mathrm{r}}^{V z}$, where $\zeta_{z}$ is the normalized molecular velocity in the $z$ direction $\left(\zeta_{z}=\xi_{z} / \sqrt{2 R^{V} T_{L}}\right), \hat{f}_{\mathrm{e}}^{V z}$ is the normalized velocity distribution function of evaporating molecules in the $z$ direction, and $\hat{f}_{\mathrm{r}}^{V z}$ is the normalized velocity distribution function of reflecting molecules in the $z$ direction, respectively. Black line in each figure denotes the normalized Maxwellian, $\hat{f}^{*}$, at $85 \mathrm{~K}$ :

$$
\zeta_{z} \hat{f}^{*}=2 \zeta_{z} \exp \left(-\zeta_{z}^{2}\right)
$$

These figures show that, for vapor molecules, the mean velocity of evaporating molecules becomes higher than that of the Maxwellian, despite the difference of $\phi$, as shown in Fig. 7(a). While, for Fig. 7(b), the mean velocity of reflecting molecules decreases. These tendencies for evaporating and reflecting molecules agree with the previous MD simulation for single component system [Tsuruta et al. (1999); Kobayashi et al. (2016b)]. However, the detailed mechanism of the deviation has not been clarified yet. Also, from these results, we cannot investigate the detailed dependency of $\phi$ for $\zeta_{z} \hat{f}_{\mathrm{e}}^{V z}$ and $\zeta_{z} \hat{f}_{\mathrm{r}}^{V z}$, because of the stochastic errors. More detailed investigation is needed to clarify the behavior of evaporating and reflecting vapor molecules.

Figures 7(c) and (d) show the velocity distribution functions of degassing and reflecting, respectively, for Ne molecules (gas) with various $\phi \mathrm{s}$. The gas constant of NC gas is used to normalized the molecular velocity. These figures show that the mean velocity of reflecting molecules takes almost the same value as that of the Maxwellian, and mean velocity of degassing molecules is slightly higher than that of the Maxwellian. These results imply that the translational motion of the reflecting Ne molecules relax more than that of the reflecting $\mathrm{Ar}$ molecules at the interface; this is due to the formation of an adsorption film of Ne molecules at the interphase (see Fig. 5(a) and (b)); once the Ne molecules move into the interphase from the gas phase, these molecules are captured in the interphase and form the adsorbed film. We investigate the stall time of reflecting $\mathrm{Ar}$ and Ne molecules in interphase as shown in Figs. 8(a) and (b). Stall time is defined as the time of reflecting molecules remained in the interphase. In these figures, the abscissa is the stall time of reflecting molecules and the ordinate is the normalized number of reflecting molecules. From this figure, we can see that the stall time of $\mathrm{NC}$ gas molecules much longer than 
that of vapor molecules; the NC gas molecules are captured in interphase as the adsorbed film for a long duration. As a result, translational motion of $\mathrm{Ne}$ molecules easily relax with the bulk liquid molecules. Also, we investigated the reflecting position of vapor and $\mathrm{NC}$ gas molecules in the interphase. The detailed are shown in Ref. [Kobayashi et al. (2016a)]

Next, we show the velocity distribution functions of evaporating and reflecting molecules tangential to the interface ( $x$ or $y$ direction) at the gas boundary $(\phi=0.021)$. Figure $9(\mathrm{a})$ is the velocity distribution function of evaporating molecules and (b) is that of the reflecting molecules. The black line is the Maxwellian at the liquid temperature. From theses figure, these functions of evaporating and reflecting molecules agree well with the Maxwellian. This tendency was also shown in the previous papers [Tsuruta et al. (1999); Kobayashi et al. (2016b)]. Hence, we can confirm that the deviation from the Maxwellian occurs only in the normal direction to the interface. The investigation of this detailed mechanism is the future work.

Figure 10 shows the velocity distribution function of condensing vapor molecules and dissolving $\mathrm{NC}$ gas molecules normal to the interface at the gas boundary. Figure 10(a) shows the velocity distribution function for condensing vapor molecules and (b) shows that of dissolving $\mathrm{NC}$ gas molecules, respectively. From Fig. 10(a), we can see that the condensing vapor molecules slightly deviate from the Maxwellian at the liquid temperature: the average speed of molecules becomes faster than the Maxwellian. Same tendency is observed in (b) for dissolving NC gas molecules. To investigate the molecular velocity dependence of the condensation coefficient, the nonequilibrium MD simulation during net evaporation or condensation is needed. For example, we showed the condensation coefficient slightly increases as the colliding molecules becomes larger during net condensation by the simulation of the Enskog-Vlasov equation [Kon et al. (2014)]. This result indicates that the condensation coefficient is dependent on the molecular velocity normal to the interface. However, to investigate the dependence of the molecular velocity on the condensation coefficient, detailed analysis is needed by treating each molecular behaviors.

3.3 Mass fluxes and $\alpha_{\mathrm{e}}^{V}$ and $\alpha_{\mathrm{e}}^{G}$ in Eqs. (5) and (6)

Figure 11 shows the mass fluxes, $J_{\mathrm{e}}^{V}, J_{\mathrm{r}}^{V}, J_{\mathrm{e}}^{G}$, and $J_{\mathrm{r}}^{G}$, at the gas interface as functions of $\phi$. These values are obtained using the method explained in section 2.3. All molecular mass fluxes are listed in Tables 1, 2, 3, and 4. Figures 11(a) and (b) are the results of Ar molecules, and Figs. 11(c) and (d) are those of Ne molecules.

For the evaporating vapor molecular mass flux $J_{\mathrm{e}}^{V}, J_{\mathrm{e}}^{V}$ at $95 \mathrm{~K}$ takes a larger value than that at $85 \mathrm{~K}$, which is due to the increase in vapor density with increasing temperature, as shown in Fig. 6. Additionally, the value of $J_{\mathrm{e}}^{V}$ decreases with increasing of $\phi$; while, $J_{\mathrm{r}}^{V}$ increases. Later, we discuss the relationship between the increase in $\phi$ and the decrease in $J_{\mathrm{e}}^{V}$. 
The degassing and reflecting NC gas molecular mass fluxes, $J_{\mathrm{e}}^{G}$ and $J_{\mathrm{r}}^{G}$, increase with increasing $\phi$. In this simulation, as $\phi$ increases, the number of $\mathrm{Ne}$ molecules increases in the simulation system and the Ne gas density increases: this is due to the increase in the mass fluxes of Ne molecules.

Once the molecular mass fluxes are obtained, we can determine the value of evaporation and condensation coefficients using the definitions in Eq. (10). Figure 12 shows the evaporation and condensation coefficients of $\mathrm{Ar}$ and $\mathrm{Ne}$ as functions of $\phi$. Figure 12(a) shows $\alpha_{\mathrm{e}}^{V}$ and $\alpha_{\mathrm{c}}^{V}$, and Figure 12(b) shows $\alpha_{\mathrm{e}}^{G}$ and $\alpha_{\mathrm{c}}^{G}$ at $85 \mathrm{~K}$ and $95 \mathrm{~K}$, respectively.

These figures show that the evaporation and condensation coefficients for Ar and Ne molecules decrease with the increase of $\phi$; we can confirm that the present method can evaluate the influence of non-condensable molecules on vapor phase changes. Additionally, evaporation and condensation coefficients take the same value for each component at same temperature; thus, we can also evaluate that the numerical system reaches the equilibrium state (see Eq. (11)). Furthermore, these values for Ar molecules become larger than those for $\mathrm{Ne}$ molecules. This is due to the small amount of dissolving Ne molecules in bulk liquid (see Fig. (5)).

The decrease of $\alpha_{\mathrm{e}}^{V}$ is due to the decrease of $J_{\mathrm{e}}^{V}$ caused by the collision of molecules in the interphase. In general, the molecular collision rate is a liner function of the number density at each position [Bird (1994)]: number of collisions increases as the number density increases; as the results, $J_{\mathrm{e}}^{V}$ decreases in interphase with the increase of $\phi$, and then, the value of $\alpha_{\mathrm{e}}^{V}$ decreases. To clarify the influence of collisions in the interphase, we investigate the number density using reflecting liquid molecules of Ar at the liquid boundary, as shown in Fig. 13(a). The reflecting liquid molecules are defined as Ar molecules that come from liquid phase and then reflect in the interphase after collision. This reflecting liquid molecules at the liquid boundary are also discussed in Gu et al. (2010b). We also investigate the reflecting positions of the Ar molecules in the vicinity of the liquid boundary within the interphase. The reflecting position is defined as the maximum arrival position in the $z$ direction of reflected liquid Ar molecules in the interphase.

Figure 13(b) shows the reflecting position of the Ar molecules in the vicinity of the liquid boundary. The abscissa is the normalized distance and the ordinate is normalized by the total number of reflected liquid molecules. This figure shows that more than $80 \%$ of the reflecting liquid molecules are reflected in the vicinity of the liquid boundary in the range of $-1.0<\bar{z}<-0.5$. Additionally, we confirm that this tendency does not change, despite the differences in liquid temperature and the value of $\phi$. Using this result, we can define the collision region of evaporating molecules and roughly estimate the length of the region in the $z$ direction from $-0.5<\bar{z}<1.0$.

Figure 13(c) shows the normalized number density of the gas mixture in the collision region shown in Fig. 13(b). The number density is normalized by that at $\phi=0$. This figure shows that the normalized number density increases with an increase in $\phi$. Furthermore, the increase tendency coincides, despite the difference in the liquid temperature. Because of molecular collisions in 
the interphase, normalized $J_{\mathrm{e}}^{V}$ decreases monotonically due to the increase of reflecting liquid Ar molecules as shown Fig. 13(d). This result indicates that one of the reason for the decrease in $\alpha_{\mathrm{e}}^{V}$ is the increase in the collision rate inside the interphase.

\subsection{KBCs for binary mixture}

From the MD simulation results, we can determine the KBCs of Eqs. (5) and (6) knowing only $J_{\mathrm{e}}^{V}, J_{\mathrm{c}}^{V}, J_{\text {coll }}^{V}, J_{\mathrm{e}}^{G}, J_{\mathrm{c}}^{G}$, and $J_{\text {coll }}^{G}$ to determine the values of $\alpha_{\mathrm{e}}^{V}, \alpha_{\mathrm{e}}^{G}, \alpha_{\mathrm{c}}^{V}$, and $\alpha_{\mathrm{c}}^{G}$, and also the values of $\rho^{V *}$ and $\rho^{G *}$ when $\hat{f}_{\text {out }}^{V}$ and $\hat{f}_{\text {out }}^{G}$ are assumed to be the normalized Maxwellian in a vapor-liquid equilibrium or weak non-equilibrium state.

On the other hand, the previous sections have shown that $\hat{f}_{\mathrm{e}}^{V z}, \hat{f}_{\mathrm{r}}^{V z}, \hat{f}_{\mathrm{e}}^{G z}$, and $\hat{f}_{\mathrm{r}}^{G z}$ deviate from the normalized Maxwell distribution at the liquid temperature. These velocity distribution functions are originated from Eqs. (8) and (9). Some studies have introduced the accommodation coefficient to discuss the deviation in the single-component case, and treat the $\mathrm{KBC}$ as a linear combination of $f_{\mathrm{e}}^{V}$ and $f_{\mathrm{r}}^{V}$ [Tsuruta et al. (2011)]. However, if $f_{\mathrm{e}}^{V}, f_{\mathrm{r}}^{V}, f_{\mathrm{e}}^{G}$, and $f_{\mathrm{r}}^{G}$ are combined into one term, as in Eqs. (5) and (6), the analysis of the Boltzmann equation will be facilitated. Hereafter, we show the relation between Eqs. (5) and (8), and Eqs. (6) and (9).

From Eqs. (8) and (9), we obtain the following mass flux relations using the definitions of the molecular mass fluxes:

$$
\begin{gathered}
\int_{\xi_{z}>0} \xi_{z} f_{\text {out }}^{V} \mathrm{~d} \boldsymbol{\xi}=J_{\text {out }}^{V}, \int_{\xi_{z}>0} \xi_{z} f_{\mathrm{e}}^{V} \mathrm{~d} \boldsymbol{\xi}=J_{\mathrm{e}}^{V} \\
\int_{\xi_{z}>0} \xi_{z} f_{\mathrm{r}}^{V} \mathrm{~d} \boldsymbol{\xi}=J_{\mathrm{r}}^{V}, \int_{\xi_{z}>0} \xi_{z} f_{\text {out }}^{G} \mathrm{~d} \boldsymbol{\xi}=J_{\text {out }}^{G} \\
\int_{\xi_{z}>0} \xi_{z} f_{\mathrm{e}}^{G} \mathrm{~d} \boldsymbol{\xi}=J_{\mathrm{e}}^{G}, \quad \int_{\xi_{z}>0} \xi_{z} f_{\mathrm{r}}^{G} \mathrm{~d} \boldsymbol{\xi}=J_{\mathrm{r}}^{G} .
\end{gathered}
$$

where $\int_{\xi_{z}>0} \mathrm{~d} \boldsymbol{\xi}$ denotes $\int_{0}^{\infty} \int_{-\infty}^{\infty} \int_{-\infty}^{\infty} \mathrm{d} \xi_{x} \mathrm{~d} \xi_{y} \mathrm{~d} \xi_{z}$. Additionally, we obtain

$$
J_{\text {out }}^{V}=J_{\mathrm{e}}^{V}+J_{\mathrm{r}}^{V}, \quad J_{\text {out }}^{G}=J_{\mathrm{e}}^{G}+J_{\mathrm{r}}^{G}
$$

where, when $\hat{f}_{\text {out }}^{V z}$ and $\hat{f}_{\text {out }}^{G z}$ are the Maxwellian, $J_{\text {out }}^{V}$ and $J_{\text {out }}^{G}$ are written as

$$
J_{\text {out }}^{V}=\rho_{\text {out }}^{V} \sqrt{R^{V} T_{L} / 2 \pi}, \quad J_{\text {out }}^{G}=\rho_{\text {out }}^{G} \sqrt{R^{G} T_{L} / 2 \pi} .
$$

The collision molecular mass fluxes, $J_{\text {coll }}^{V}$ and $J_{\text {coll }}^{G}$, are obtained using Eqs. (2) and (4):

$$
J_{\text {coll }}^{V}=\sigma^{V} \sqrt{\frac{R^{V} T_{L}}{2 \pi}}, \quad J_{\text {coll }}^{G}=\sigma^{G} \sqrt{\frac{R^{G} T_{L}}{2 \pi}} .
$$


Using Eqs. (21) and (22), Eq. (20) can be rewritten as

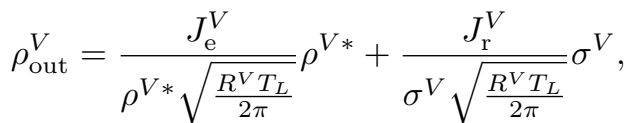

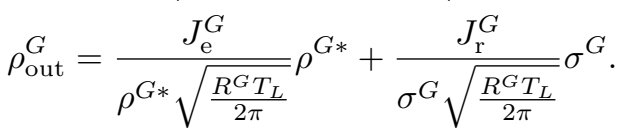

Using the definitions of $\alpha_{\mathrm{e}}^{V}, \alpha_{\mathrm{c}}^{V}, \alpha_{\mathrm{e}}^{G}, \alpha_{\mathrm{c}}^{G}$ (see Eq. (10)), $J_{\text {coll }}^{V}=J_{\mathrm{c}}^{V}+J_{\mathrm{r}}^{V}$ and $J_{\text {coll }}^{G}=J_{\mathrm{c}}^{G}+J_{\mathrm{r}}^{G}$, we obtain the densities $\rho_{\text {out }}^{V}$ and $\rho_{\text {out }}^{G}$ as

$$
\begin{gathered}
\rho_{\text {out }}^{V}=\alpha_{\mathrm{e}}^{V} \rho^{V *}+\left(1-\alpha_{\mathrm{c}}^{V}\right) \sigma^{V}, \\
\rho_{\text {out }}^{G}=\alpha_{\mathrm{e}}^{G} \rho^{G *}+\left(1-\alpha_{\mathrm{c}}^{G}\right) \sigma^{G} .
\end{gathered}
$$

Thus, as shown in Eqs. (25) and (26), we can obtain Eqs. (5) and (6) from Eqs. (8) and (9). From this derivation, the deviations of the velocity distribution functions, $f_{\mathrm{e}}^{V}, f_{\mathrm{r}}^{V}, f_{\mathrm{e}}^{G}$, and $f_{\mathrm{r}}^{G}$, from the normalized Maxwell distribution are included in the evaporation and condensation coefficients.

The position of the gas boundary is the KBC for the Boltzmann equation $(\bar{z}=3.0$ in this study). As shown in a previous MD simulation [Kobayashi et al. (2016b)], the values of these mass fluxes change based on the position of the liquid boundaries, which leads to changes in the values of evaporation and condensation coefficients. However, $J_{\text {out }}^{V}$ and $J_{\text {out }}^{G}$ have constant values, and the functional form of Eqs. (5) and (6) do not change. Therefore, even if we choose the other position of the liquid boundary, the correct values of mass, momentum, and energy fluxes are obtained from the KBCs, $f_{\text {out }}^{V}$ and $f_{\text {out }}^{G}$.

In this study, we proposed new KBCs for the Ar-Ne binary mixture problem in equilibrium. Using the same procedure, we can create KBCs for nonequilibrium states of the binary mixture.

\section{Conclusion}

In the present study, we used MD simulation to investigate the KBCs of the binary mixture in equilibrium. We utilized $\mathrm{Ar}$ as vapor molecules and $\mathrm{Ne}$ as non-condensable gas (NC gas) molecules. We investigated the molecules behaviors utilizing the improved two-boundary method proposed in our previous study (Kobayashi et al. (2016b)), which allows us to easily count the evaporating, condensing, degassing, dissolving, and reflecting molecules, and also to accurately evaluate the velocity distribution function of the KBCs at the interface.

The present results confirmed that the mean velocity of the evaporating vapor molecules and degassing $\mathrm{NC}$ gas molecules in the direction normal to the interface were slightly higher than that of the normalized Maxwell distribution at the liquid temperature. For vapor molecules, this result agrees with the previous MD simulations. Additionally, we found that the evaporation and 
condensation coefficients for vapor and NC gas molecules decrease with an increase of molar fraction of NC gas molecules in the liquid. From the present results, we conclude that the KBCs for binary mixture can be constructed as functions of the molar fraction and liquid temperature.

\section{Acknowledgments}

This work was supported by JSPS KAKENHI Grant Number 16K06064. Many people, especially Dr. H. Yaguchi (National institute of technology, gunma college), Mr. T. Yahagi, and Mr. K. Hori, have made valuable comments and suggestions.

\section{References}

K. Aoki, S. Takata, S. Kosuge, Vapor flows caused by evaporation and condensation on two parallel plane surfaces: Effect of the presence of a noncondensable gas. Physics of Fluids (1994-present) 10(6), 1519-1533 (1998)

V.G. Baidakov, S.P. Protsenko, Molecular-dynamics investigation of phase equilibrium and surface tension in argon- neon system. The Journal of Physical Chemistry C 112(44), 17231-17234 (2008)

G.A. Bird, Molecular gas dynamics and the direct simulation of gas flows (1994)

S. Cheng, J.B. Lechman, S.J. Plimpton, G.S. Grest, Evaporation of lennard-jones fluids. The Journal of chemical physics 134(22), 224704 (2011)

A. Frezzotti, Boundary conditions at the vapor-liquid interface. Physics of Fluids (1994present) 23(3), 030609 (2011a)

A. Frezzotti, Non-equilibrium structure of the vapor-liquid interface of a binary fluid, in AIP Conference Proceedings-American Institute of Physics, vol. 1333, 2011b, p. 161

K. Gu, C.B. Watkins, J. Koplik, Molecular dynamics simulation of the equilibrium liquidvapor interphase with solidification. Fluid Phase Equilibria 297(1), 77-89 (2010a)

K. Gu, C.B. Watkins, J. Koplik, Multiscale molecular simulations of argon vapor condensation onto a cooled substrate with bulk flow. Physics of Fluids (1994-present) 22(11), $112002(2010 b)$

J.-P. Hansen, I.R. McDonald, Theory of simple liquids (1990)

T. Ishiyama, T. Yano, S. Fujikawa, Molecular dynamics study of kinetic boundary condition at an interface between argon vapor and its condensed phase. Physics of Fluids (1994present) 16(8), 2899-2906 (2004)

K. Kobayashi, K. Sasaki, M. Kon, H. Fujii, M. Watanabe, Molecular dynamics simulation on kinetic boundary conditions of gas-vapor binary mixture, in AIP Conference Proceedings-American Institute of Physics, submitted, 2016a

K. Kobayashi, K. Hori, M. Kon, K. Sasaki, M. Watanabe, Molecular dynamics study on evaporation and reflection of monatomic molecules to construct kinetic boundary condition in vapor-liquid equilibria. Heat and Mass Transfer 52(9), 1851-1859 (2016b). doi:10.1007/s00231-015-1700-6. http://dx.doi.org/10.1007/s00231-015-1700-6

M. Kon, K. Kobayashi, M. Watanabe, Method of determining kinetic boundary conditions in net evaporation/condensation. Physics of Fluids (1994-present) 26(7), 072003 (2014)

M. Kon, K. Kobayashi, M. Watanabe, Liquid temperature dependence of kinetic boundary condition at vapor-liquid interface. International Journal of Heat and Mass Transfer 99, 317-326 (2016a)

M. Kon, K. Kobayashi, M. Watanabe, Molecular simulation of evaporation mass flux during net evaporation/condensation, in AIP Conference Proceedings-American Institute of Physics, submitted, 2016b 
W. Kreider, L.A. Crum, M.R. Bailey, O.A. Sapozhnikov, A reduced-order, single-bubble cavitation model with applications to therapeutic ultrasound. The Journal of the Acoustical Society of America 130(5), 3511-3530 (2011)

A. Kryukov, V.Y. Levashov, Boundary conditions on the vapor liquid interface at strong condensation. Heat and Mass Transfer, 1-9 (2015)

J. Lee, T. Laoui, R. Karnik, Nanofluidic transport governed by the liquid/vapour interface. Nature nanotechnology 9(4), 317-323 (2014)

Z. Lu, S. Narayanan, E.N. Wang, Modeling of evaporation from nanopores with nonequilibrium and nonlocal effects. Langmuir 31(36), 9817-9824 (2015)

Y. Matsumoto, F. Takemura, Influence of internal phenomena on gas bubble motion. effects of thermal diffusion, phase change on the gas-liquid interface and mass diffusion between vapor and noncondensable gas in the collapsing phase. JSME International Journal Series B 37(2), 288-296 (1994)

R. Meland, A. Frezzotti, T. Ytrehus, B. Hafskjold, Nonequilibrium molecular-dynamics simulation of net evaporation and net condensation, and evaluation of the gas-kinetic boundary condition at the interphase. Physics of Fluids (1994-present) 16(2), 223-243 (2004)

W. Streett, Liquid-vapor equilibrium in the system neon-argon. The Journal of Chemical Physics 42(2), 500-503 (1965)

W. Streett, Liquid-vapor phase behavior and liquid phase density in the system neon-argon at high pressures. The Journal of Chemical Physics 46(9), 3282-3286 (1967)

S. Taguchi, K. Aoki, S. Takata, Vapor flows condensing at incidence onto a plane condensed phase in the presence of a noncondensable gas. i. subsonic condensation. Physics of Fluids (1994-present) 15(3), 689-705 (2003)

S. Takata, S. Yasuda, S. Kosuge, K. Aoki, Numerical analysis of thermal-slip and diffusionslip flows of a binary mixture of hard-sphere molecular gases. Physics of Fluids (1994present) 15(12), 3745-3766 (2003)

T. Tsuruta, H. Tanaka, T. Masuoka, Condensation/evaporation coefficient and velocity distributions at liquid-vapor interface. International Journal of Heat and Mass Transfer 22(42), 4107-4116 (1999)

T. Tsuruta, A. Tokunaga, G. Nagayama, Molecular boundary conditions and accommodation coefficient on a nonequilibrium liquid surface, in AIP Conference ProceedingsAmerican Institute of Physics, vol. 1333, 2011, p. 859

M. Wörner, Numerical modeling of multiphase flows in microfluidics and micro process engineering: a review of methods and applications. Microfluidics and nanofluidics 12(6), 841-886 (2012)

J.-F. Xie, S.S. Sazhin, B.-Y. Cao, Molecular dynamics study of condensation/evaporation and velocity distribution of $\mathrm{n}$-dodecane at liquid-vapour phase equilibria. Journal of Thermal Science and Technology 7(1), 288-300 (2012) 
Table 1 MD simulation results of Ar molecules at $85 \mathrm{~K}$.

\begin{tabular}{ccccccccc}
\hline $\begin{array}{c}\phi \\
{[-]}\end{array}$ & $\begin{array}{c}J_{\text {out }^{2}}^{V} \\
{\left[\mathrm{~g} /\left(\mathrm{cm}^{2} \mathrm{~s}\right)\right]}\end{array}$ & $\begin{array}{c}J_{\mathrm{coll}_{2}}^{V} \\
{\left[\mathrm{~g} /\left(\mathrm{cm}^{2} \mathrm{~s}\right)\right]}\end{array}$ & $\begin{array}{c}J_{\mathrm{e}}^{V} \\
{\left[\mathrm{~g} /\left(\mathrm{cm}^{2} \mathrm{~s}\right)\right]}\end{array}$ & $\begin{array}{c}J_{\mathrm{r}}^{V} \\
{\left[\mathrm{~g} /\left(\mathrm{cm}^{2} \mathrm{~s}\right)\right]}\end{array}$ & $\begin{array}{c}J_{\mathrm{c}}^{V} \\
{\left[\mathrm{~g} /\left(\mathrm{cm}^{2} \mathrm{~s}\right)\right]}\end{array}$ & $\begin{array}{c}\alpha_{\mathrm{e}}^{V} \\
{[-]}\end{array}$ & $\begin{array}{c}\alpha_{\mathrm{c}}^{V} \\
{[-]}\end{array}$ & $\begin{array}{c}\rho^{V *} \\
{\left[\mathrm{~kg} / \mathrm{m}^{3}\right]}\end{array}$ \\
\hline 0.000 & 26.475 & 26.468 & 23.079 & 3.079 & 23.079 & 0.872 & 0.872 & 4.955 \\
0.005 & 26.549 & 26.555 & 21.058 & 5.491 & 21.064 & 0.793 & 0.793 & 5.167 \\
0.011 & 27.508 & 27.456 & 19.831 & 7.677 & 19.780 & 0.721 & 0.720 & 5.551 \\
0.016 & 28.417 & 28.217 & 18.780 & 9.637 & 18.580 & 0.661 & 0.658 & 5.881 \\
0.021 & 30.099 & 29.943 & 18.186 & 11.913 & 18.030 & 0.604 & 0.602 & 6.272 \\
0.024 & 32.434 & 32.220 & 18.051 & 14.383 & 17.837 & 0.557 & 0.554 & 6.957 \\
\hline
\end{tabular}

Table 2 MD simulation results of Ne molecules at $85 \mathrm{~K}$.

\begin{tabular}{ccccccccc}
\hline $\begin{array}{c}\phi \\
{[-]}\end{array}$ & $\begin{array}{c}J_{\text {out }^{G}}^{G} \\
{\left[\mathrm{~g} /\left(\mathrm{cm}^{2} \mathrm{~s}\right)\right]}\end{array}$ & $\begin{array}{c}J_{\text {coll }}^{G} \\
{\left[\mathrm{~g} /\left(\mathrm{cm}^{2} \mathrm{~s}\right)\right]}\end{array}$ & $\begin{array}{c}J_{\mathrm{e}}^{G} \\
{\left[\mathrm{~g} /\left(\mathrm{cm}^{2} \mathrm{~s}\right)\right]}\end{array}$ & $\begin{array}{c}J_{\mathrm{r}}^{G} \\
{\left[\mathrm{~g} /\left(\mathrm{cm}^{2} \mathrm{~s}\right)\right]}\end{array}$ & $\begin{array}{c}J_{\mathrm{c}}^{G} \\
{\left[\mathrm{~g} /\left(\mathrm{cm}^{2} \mathrm{~s}\right)\right]}\end{array}$ & $\begin{array}{c}\alpha_{\mathrm{e}}^{G} \\
{[-]}\end{array}$ & $\begin{array}{c}\alpha_{\mathrm{c}}^{G} \\
{[-]}\end{array}$ & $\begin{array}{c}\rho^{G *} \\
{\left[\mathrm{~kg} / \mathrm{m}^{3}\right]}\end{array}$ \\
\hline 0.005 & 177.492 & 177.499 & 14.290 & 163.201 & 14.298 & 0.081 & 0.081 & 12.347 \\
0.011 & 349.897 & 349.873 & 24.618 & 325.280 & 24.594 & 0.070 & 0.070 & 24.353 \\
0.016 & 518.642 & 518.489 & 34.187 & 484.455 & 34.034 & 0.066 & 0.066 & 37.089 \\
0.021 & 684.810 & 684.650 & 42.410 & 642.400 & 42.249 & 0.062 & 0.062 & 49.599 \\
0.024 & 850.265 & 849.941 & 48.778 & 801.487 & 48.454 & 0.057 & 0.057 & 62.258 \\
\hline
\end{tabular}

Table 3 MD simulation results of Ar molecules at $95 \mathrm{~K}$.

\begin{tabular}{ccccccccc}
\hline $\begin{array}{c}\phi \\
{[-]}\end{array}$ & $\begin{array}{c}J_{\text {out }}^{V} \\
{\left[\mathrm{~g} /\left(\mathrm{cm}^{2} \mathrm{~s}\right)\right]}\end{array}$ & $\begin{array}{c}J_{\text {coll }}^{V} \\
{\left[\mathrm{~g} /\left(\mathrm{cm}^{2} \mathrm{~s}\right)\right]}\end{array}$ & $\begin{array}{c}J_{\mathrm{e}}^{V} \\
{\left[\mathrm{~g} /\left(\mathrm{cm}^{2} \mathrm{~s}\right)\right]}\end{array}$ & $\begin{array}{c}J_{\mathrm{r}}^{V} \\
{\left[\mathrm{~g} /\left(\mathrm{cm}^{2} \mathrm{~s}\right)\right]}\end{array}$ & $\begin{array}{c}J_{\mathrm{c}}^{V} \\
{\left[\mathrm{~g} /\left(\mathrm{cm}^{2} \mathrm{~s}\right)\right]}\end{array}$ & $\begin{array}{c}\alpha_{\mathrm{e}}^{V} \\
{[-]}\end{array}$ & $\begin{array}{c}\alpha_{\mathrm{c}}^{V} \\
{[-]}\end{array}$ & $\begin{array}{c}\rho^{V *} \\
{\left[\mathrm{~kg} / \mathrm{m}^{3}\right]}\end{array}$ \\
\hline 0.000 & 65.015 & 65.016 & 49.867 & 15.148 & 49.868 & 0.767 & 0.767 & 11.909 \\
0.007 & 66.535 & 66.385 & 45.743 & 20.792 & 45.593 & 0.688 & 0.687 & 12.548 \\
0.013 & 68.154 & 68.006 & 41.950 & 26.204 & 41.803 & 0.616 & 0.615 & 13.322 \\
0.019 & 73.902 & 73.612 & 41.127 & 32.775 & 40.838 & 0.557 & 0.555 & 14.686 \\
0.026 & 75.131 & 74.796 & 38.028 & 37.103 & 37.692 & 0.506 & 0.504 & 15.618 \\
0.032 & 78.755 & 78.297 & 36.412 & 42.343 & 35.953 & 0.462 & 0.459 & 16.174 \\
\hline
\end{tabular}

Table 4 MD simulation results of Ne molecules at $95 \mathrm{~K}$.

\begin{tabular}{ccccccccc}
\hline $\begin{array}{c}\phi \\
{[-]}\end{array}$ & $\begin{array}{c}J_{\text {out }}^{G} \\
{\left[\mathrm{~g} /\left(\mathrm{cm}^{2} \mathrm{~s}\right)\right]}\end{array}$ & $\begin{array}{c}J_{\mathrm{coll}}^{G} \\
{\left[\mathrm{~g} /\left(\mathrm{cm}^{2} \mathrm{~s}\right)\right]}\end{array}$ & $\begin{array}{c}J_{\mathrm{e}}^{G} \\
{\left[\mathrm{~g} /\left(\mathrm{cm}^{2} \mathrm{~s}\right)\right]}\end{array}$ & $\begin{array}{c}J_{\mathrm{r}}^{G} \\
{\left[\mathrm{~g} /\left(\mathrm{cm}^{2} \mathrm{~s}\right)\right]}\end{array}$ & $\begin{array}{c}J_{\mathrm{c}}^{G} \\
{\left[\mathrm{~g} /\left(\mathrm{cm}^{2} \mathrm{~s}\right)\right]}\end{array}$ & $\begin{array}{c}\alpha_{\mathrm{e}}^{G} \\
{[-]}\end{array}$ & $\begin{array}{c}\alpha_{\mathrm{c}}^{G} \\
{[-]}\end{array}$ & $\begin{array}{c}\rho^{G *} \\
{\left[\mathrm{~kg} / \mathrm{m}^{3}\right]}\end{array}$ \\
\hline 0.007 & 188.263 & 188.271 & 13.962 & 174.301 & 13.970 & 0.074 & 0.074 & 12.459 \\
0.013 & 364.939 & 364.848 & 25.799 & 339.141 & 25.707 & 0.071 & 0.070 & 24.982 \\
0.019 & 549.319 & 549.149 & 36.643 & 512.677 & 36.472 & 0.067 & 0.066 & 37.503 \\
0.026 & 705.317 & 705.072 & 43.541 & 661.776 & 43.296 & 0.062 & 0.061 & 50.129 \\
0.032 & 896.437 & 895.894 & 51.759 & 844.678 & 51.216 & 0.058 & 0.057 & 62.761 \\
\hline
\end{tabular}




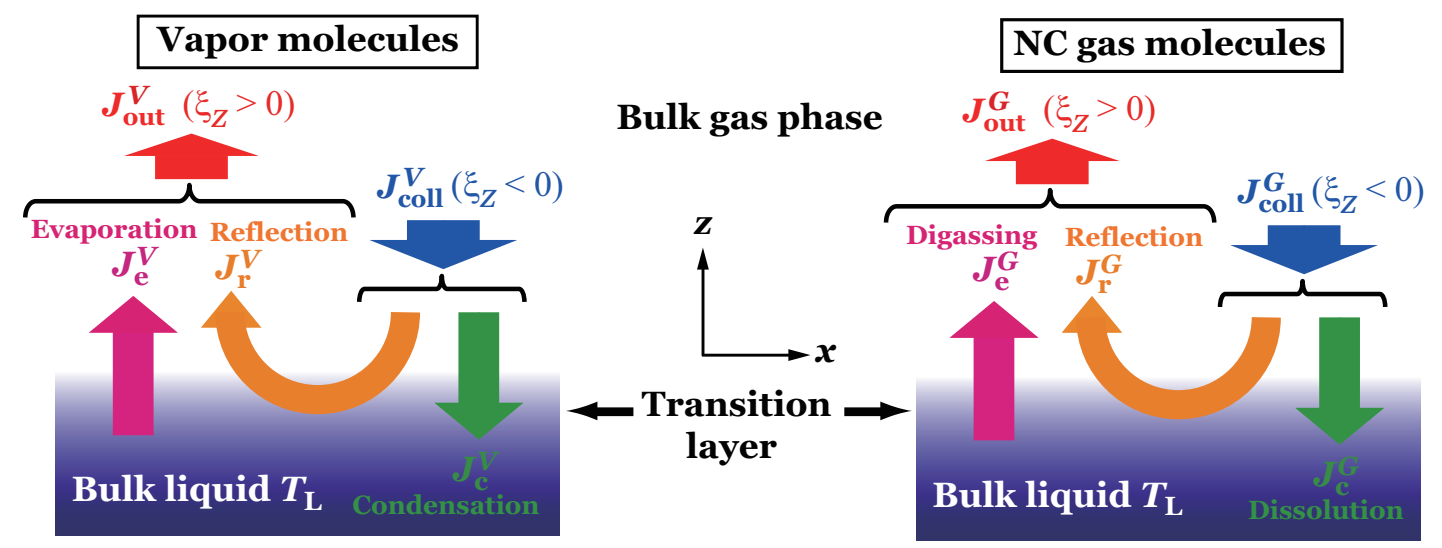

Fig. 1 Molecular mass flux relation at gas-liquid interface. Left: mass fluxes for vapor molecules, and right: mass fluxes for NC gas molecules, respectively. 


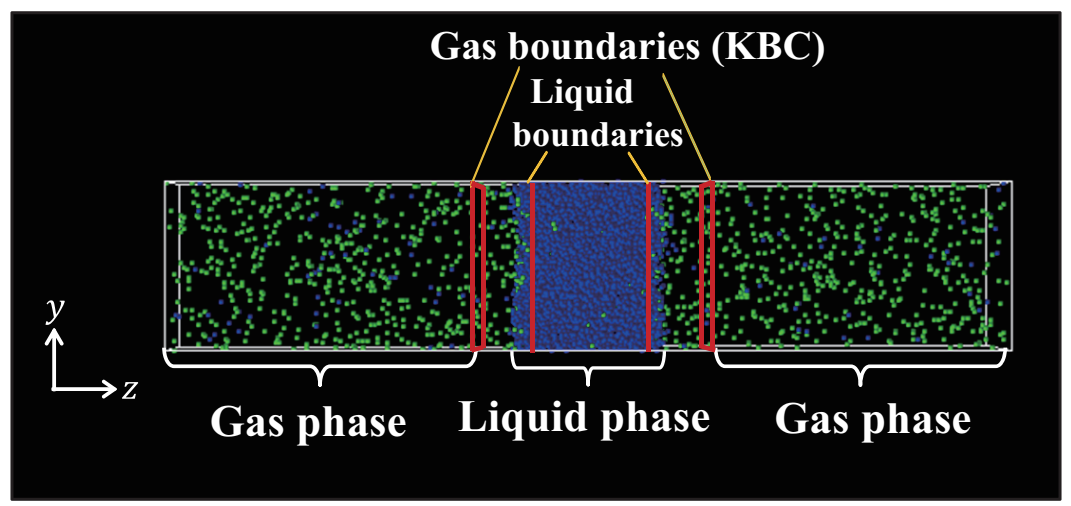

Fig. 2 MD simulation of the present study. Blue sphere denotes the vapor (Ar) molecule and green sphere denotes the $\mathrm{NC}$ gas $(\mathrm{Ne})$ molecule, respectively. 


\section{Vapor (Ar) molecules}

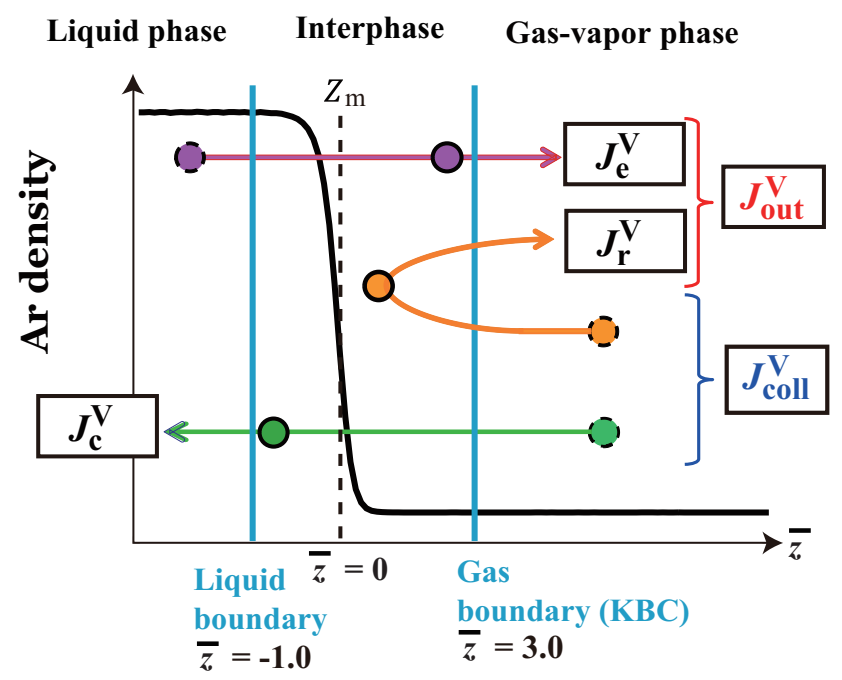

\section{NC gas (Ne) molecules}

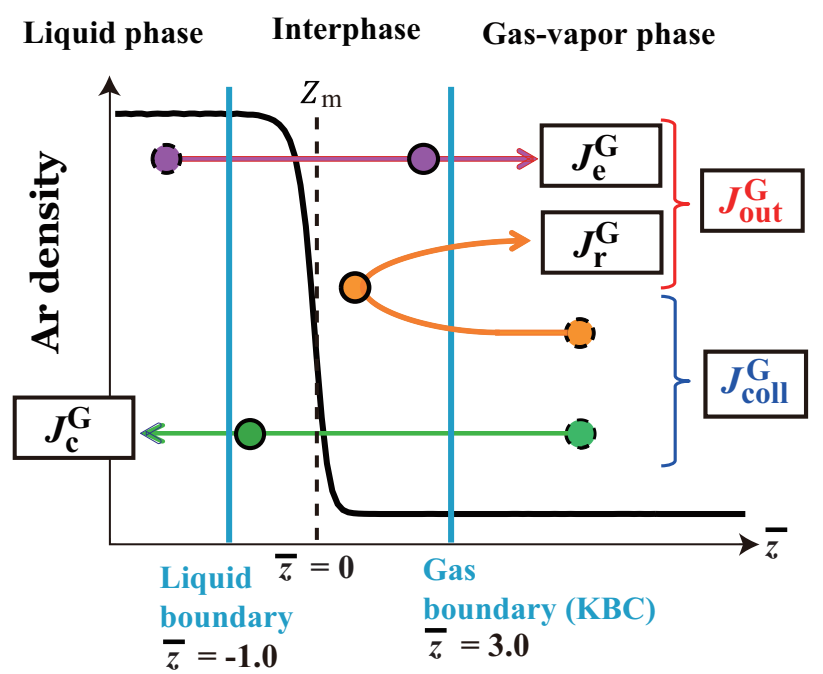

Fig. 3 Molecular mass fluxes at a gas-liquid interface. There are gas and liquid boundaries in the vicinity of the density transition layer of Ar molecules. $\bar{z}$ is the normalized $z$ axis. 


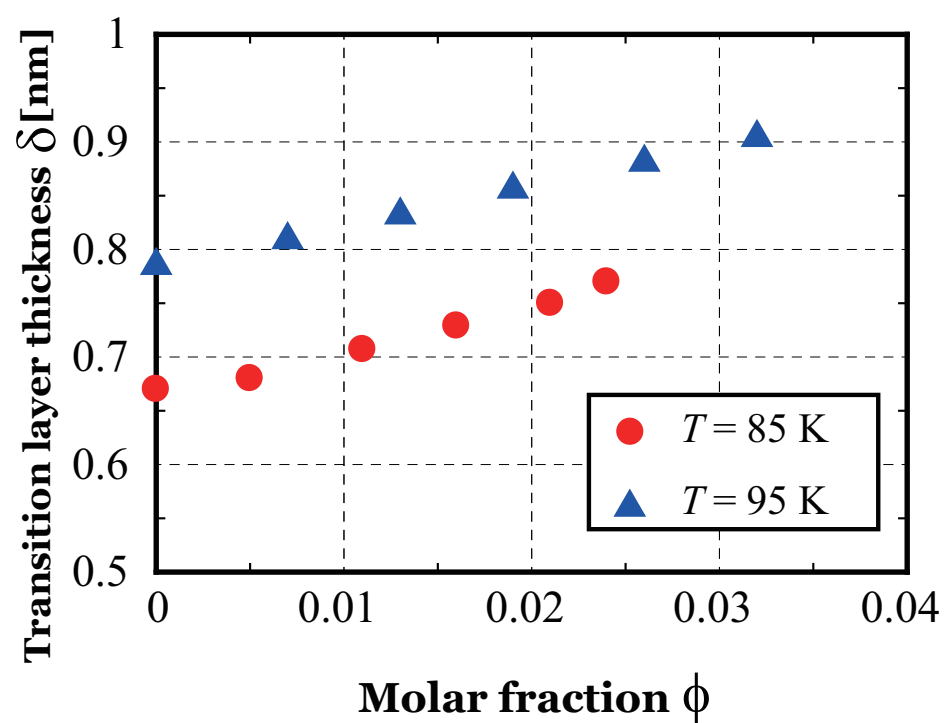

Fig. 4 Relationship between transition layer thickness $\delta$ and molar fraction $\phi$. 
(a) $T=85 \mathrm{~K}$

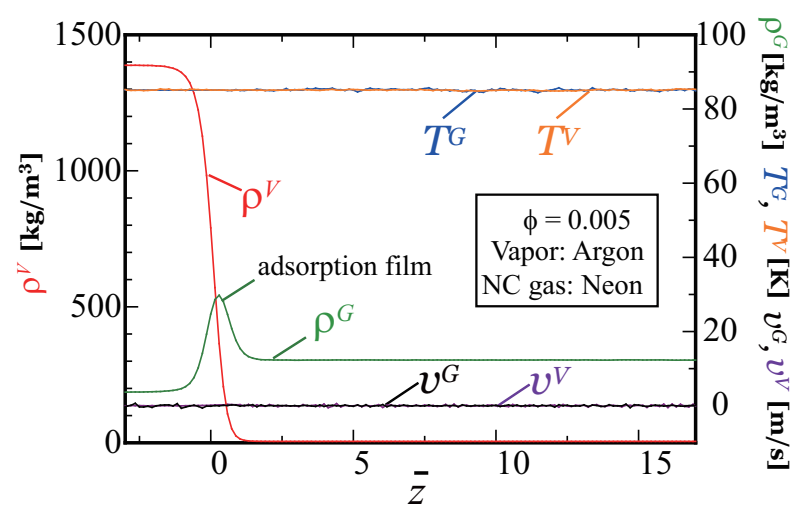

(b) $T=95 \mathrm{~K}$

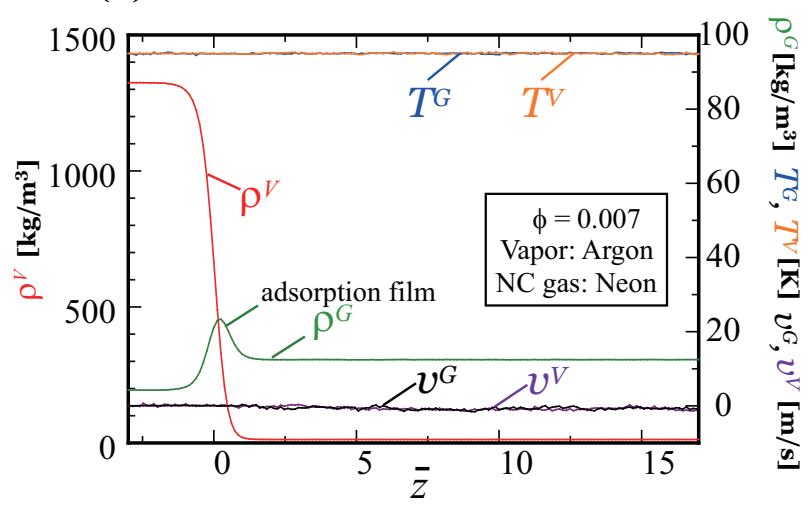

Fig. 5 Macroscopic variables of $\mathrm{Ar}$ and Ne molecules at $85 \mathrm{~K}$ or $95 \mathrm{~K}$ in the vapor-liquid equilibrium state: (a) $85 \mathrm{~K}$ and $\phi=0.005$, (b) $95 \mathrm{~K}$ and $\phi=0.007$. 

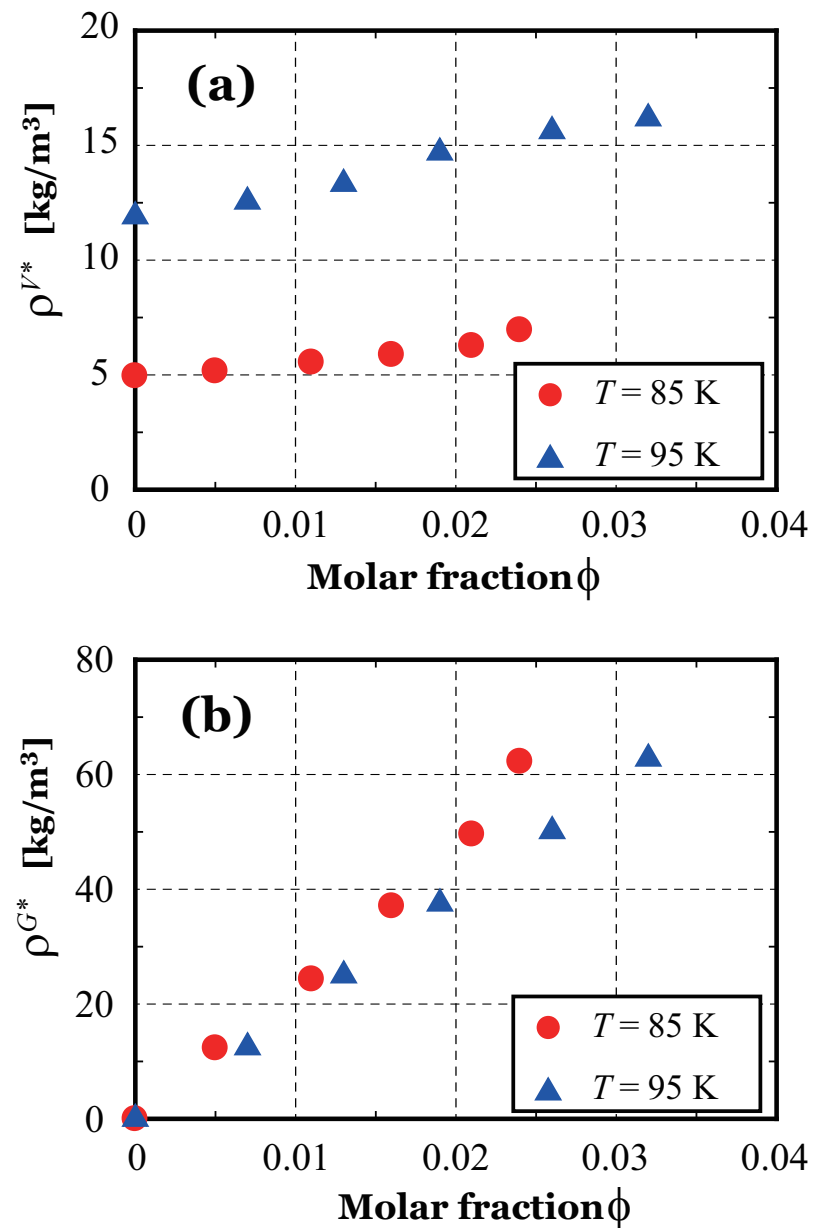

Fig. 6 Relation of vapor and NC gas densities and molar fraction $\phi$ at $85 \mathrm{~K}$ and $95 \mathrm{~K}$ : (a) saturated vapor density $\rho^{V *}$, and (a) NC gas density $\rho^{G *}$ in equilibrium. 

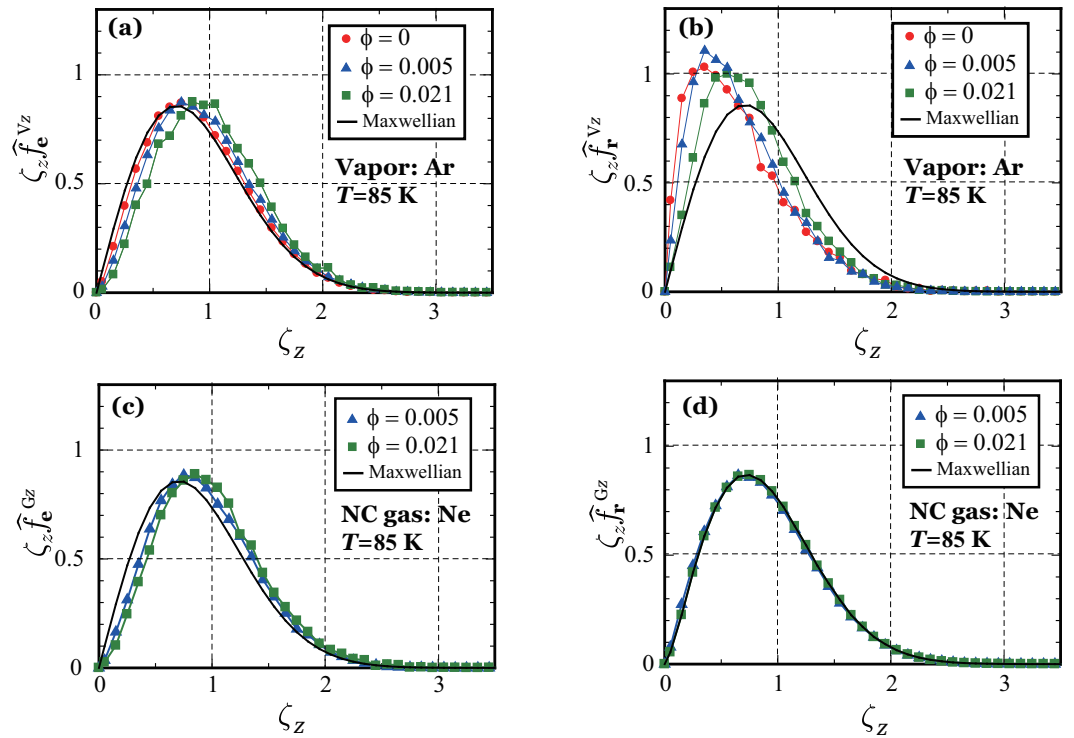

Fig. 7 Molecular velocity distribution functions for vapor and NC gas molecules: (a) $\hat{f}_{\mathrm{e}}^{V z}$ for evaporation vapor molecules in $z$ direction; (b) $\hat{f}_{\mathrm{r}}^{V z}$ for reflecting vapor molecules in $z$ direction; (c) $\hat{f}_{\mathrm{e}}^{G z}$ for degassing NC gas molecules in $z$ direction, and (d) $\hat{f}_{\mathrm{r}}^{G z}$ for reflecting $\mathrm{NC}$ gas molecules in $z$ direction, respectively.
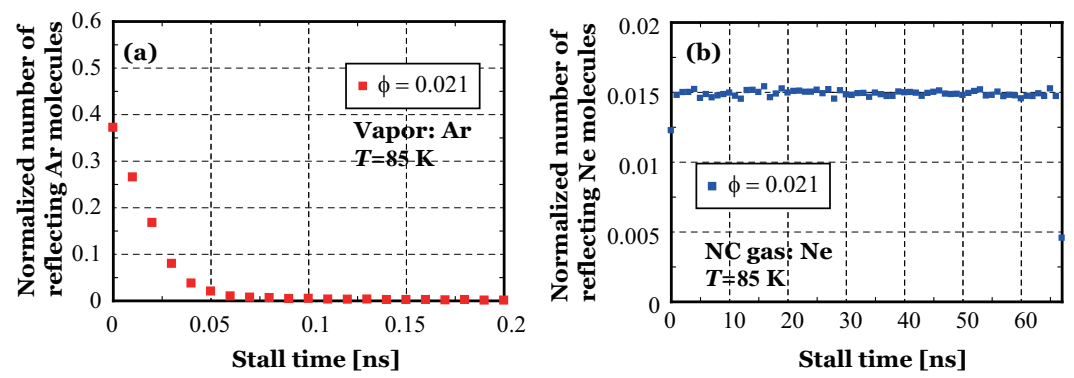

Fig. 8 Relation of stall time and normalized number of reflecitng molecules at $T=85 \mathrm{~K}$ $(\phi=0.021)$ : (a) shows the stall time for reflecting vapor molecules, and (b) shows that for reflecting gas molecules, respectively. 

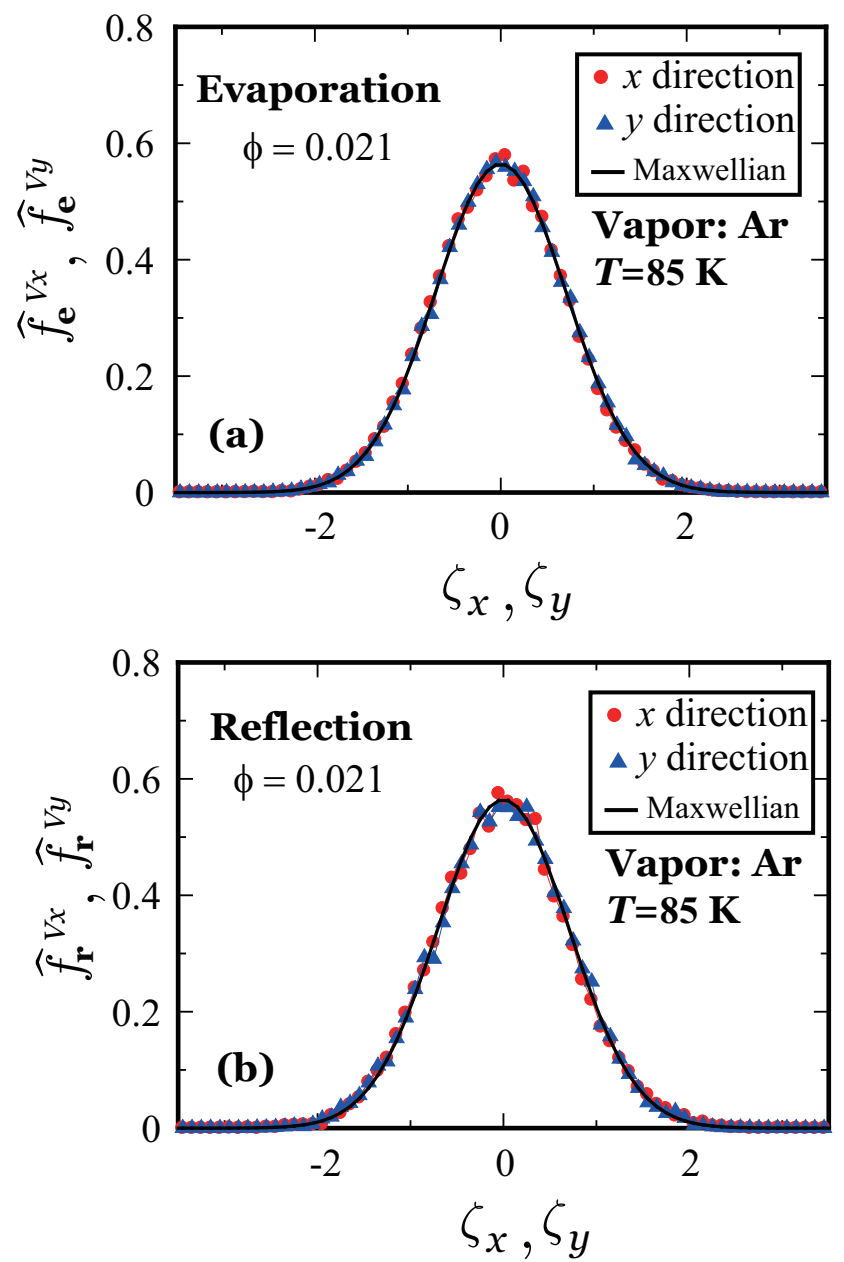

Fig. 9 Molecular velocity distribution functions of tangential component to the interface for vapor molecules at the gas boundary: (a) velocity distribution function of evaporating vapor molecules; (b) that of reflecting vapor molecules. 

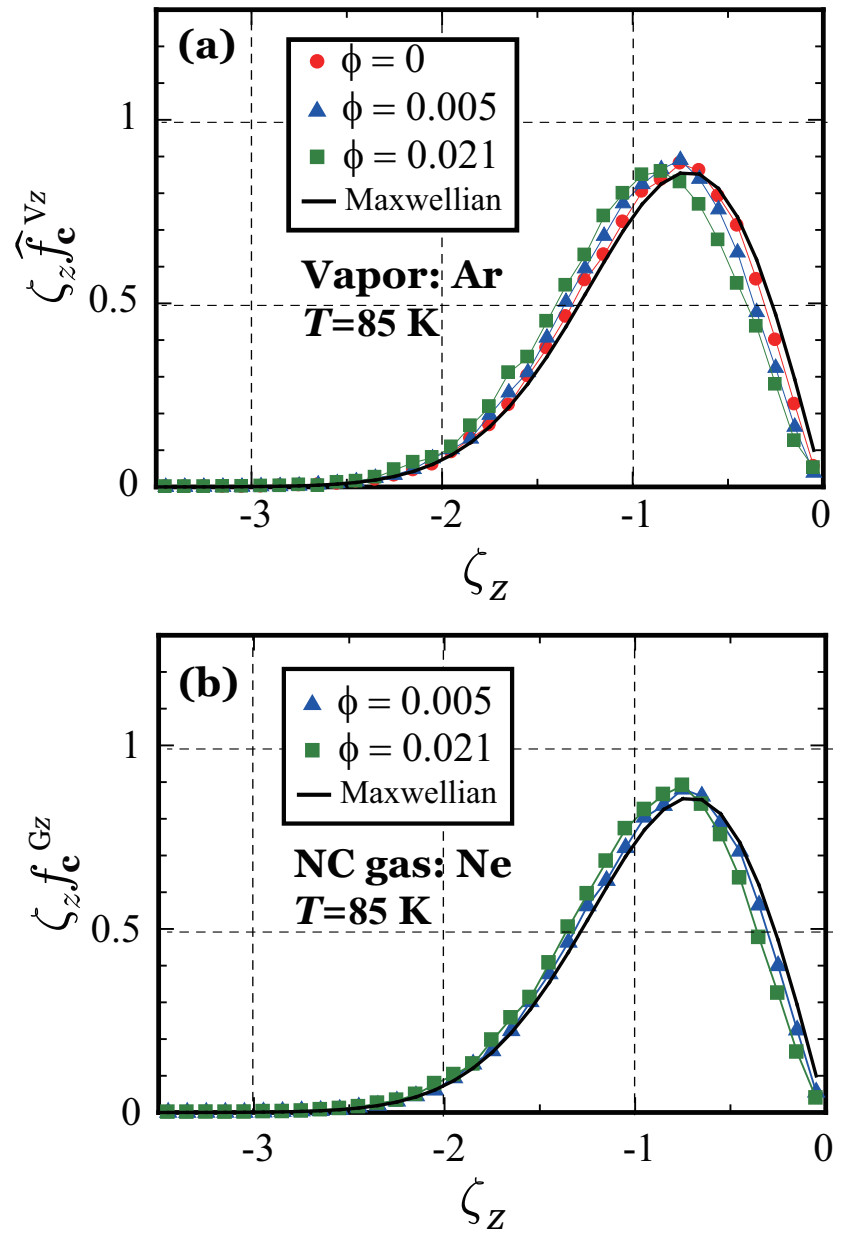

Fig. 10 Molecular velocity distribution functions of condensing vapor molecules and dissolving NC gas molecules at the gas boundary: (a) velocity distribution function of condensing vapor molecules; (b) that of dissolving NC gas molecules. 

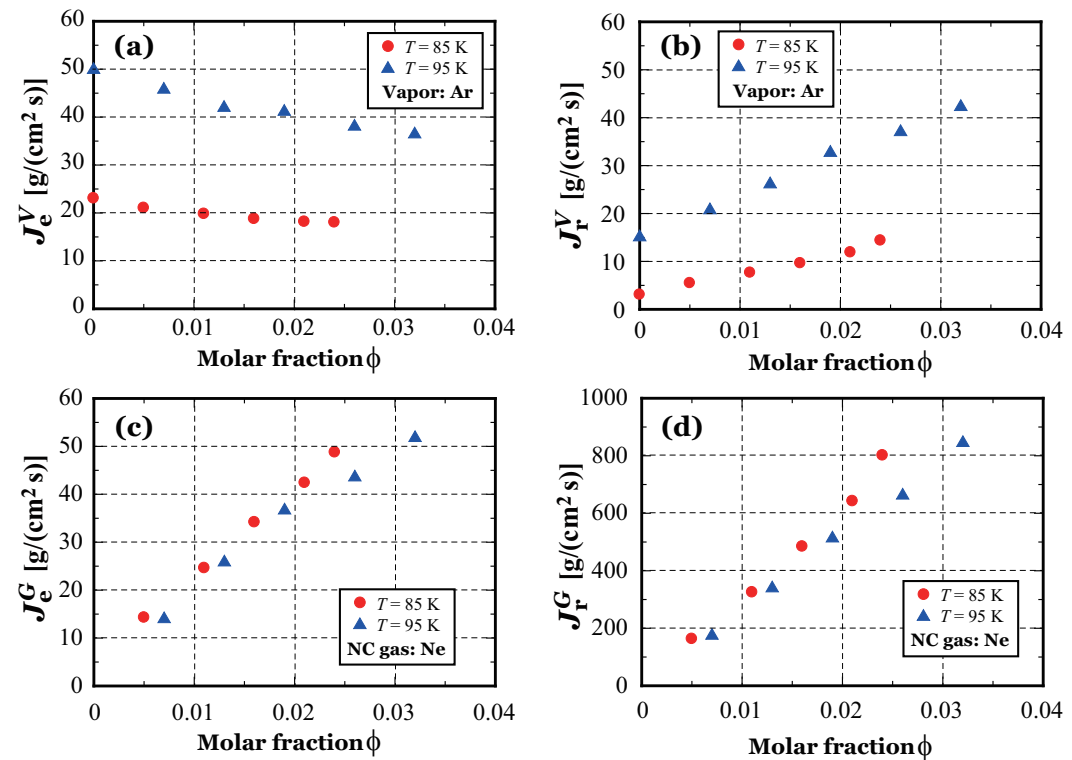

Fig. 11 Relation between mass fluxes at the gas interface and molar fraction $\phi$ at $85 \mathrm{~K}$ and $95 \mathrm{~K}$ : (a) evaporating vapor mass flux $J_{\mathrm{e}}^{V}$, (b) reflecting vapor mass flux $J_{\mathrm{r}}^{V}$, (c) degassing $\mathrm{NC}$ gas mass flux $J_{\mathrm{e}}^{G}$, and (d) reflecting $\mathrm{NC}$ gas mass flux $J_{\mathrm{r}}^{G}$.
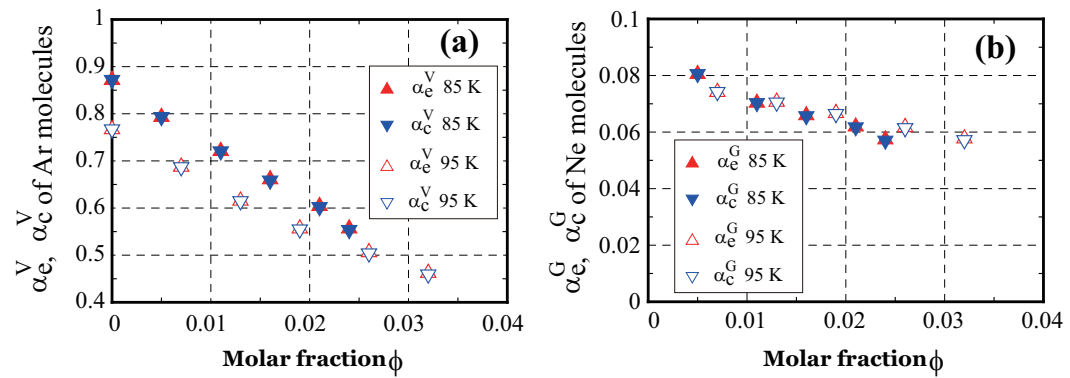

Fig. 12 Evaporation and condensation coefficients for vapor and NC gas molecules as the function of $\phi$ : (a) vapor molecules, and (b) NC gas molecules. 
Vapor (Ar) molecules
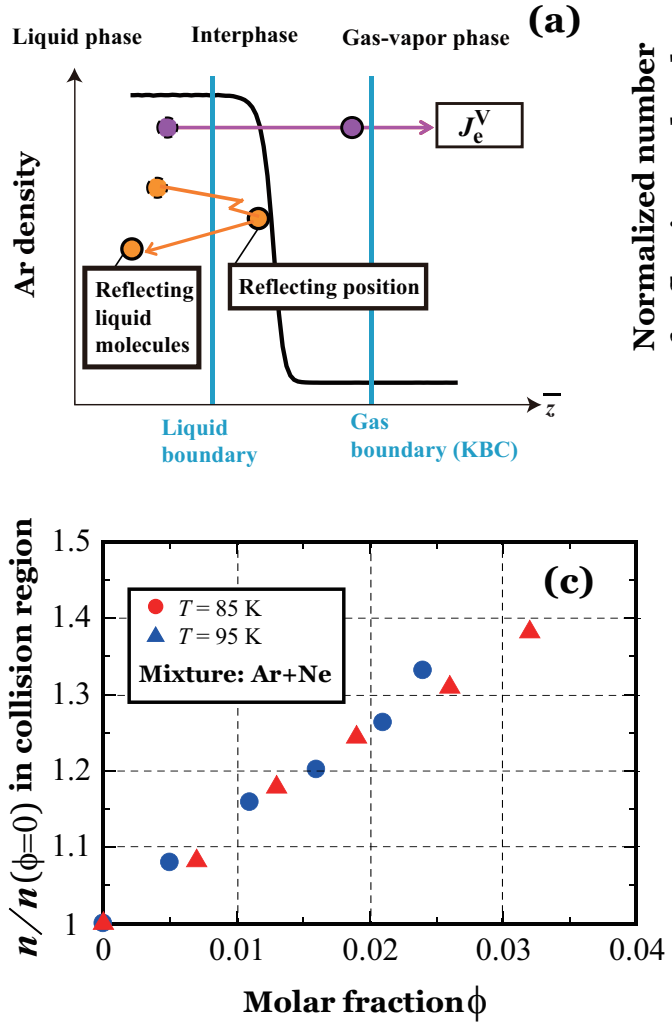

(a)
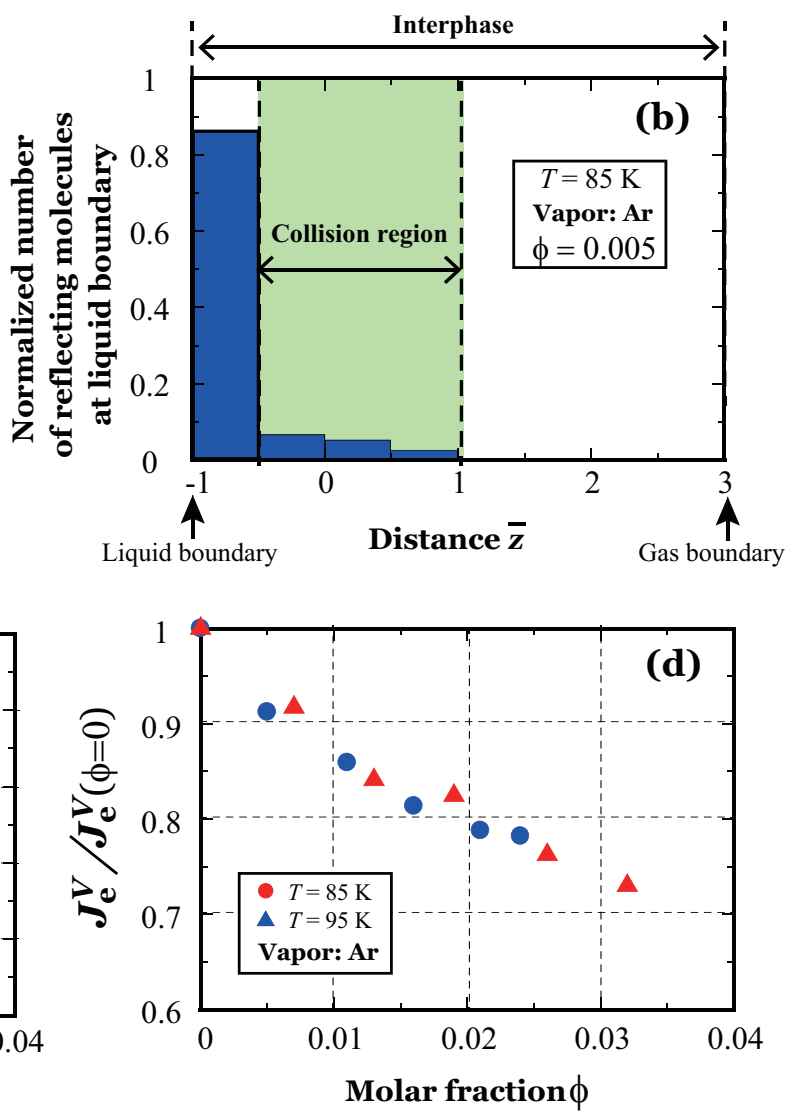

Fig. 13 Reflecting vapor molecules at liquid boundary: (a) schematic of reflecting molecules at liquid boundary; (b) reflecting position of vapor molecules at liquid boundary; (c) normalized number density of gas mixture in interphase vs. $\phi$, and (d) normalized evaporating vaopr molecular mass flux vs. $\phi$. 